\title{
Evaluation of the Clinical Efficacy of Intranasal Desmopressin Spray, Parenteral Diclofenac or Their Combination in the Treatment of Acute Renal Pain Caused by Urolithiasis
}

\author{
Ala'a Al-Deen Al-Dabbagh', Taha Kaream Kadhum², Ammar Salman Alfaiadh² \\ ${ }^{1}$ Department of Urology, University of Al-Mustansiriyah College of Medicine, Baghdad, Iraq \\ ${ }^{2}$ Department of Urology, Al-Yarmouk Teaching Hospital, Baghdad, Iraq \\ Email: alaaa1962@yahoo.co.uk, tahak967@gmail.com, al abby75@gmail.com
}

How to cite this paper: Al-Dabbagh, A.A.-D., Kadhum, T.K. and Alfaiadh, A.S. (2017) Evaluation of the Clinical Efficacy of Intranasal Desmopressin Spray, Parenteral Diclofenac or Their Combination in the Treatment of Acute Renal Pain Caused by Urolithiasis. Open Journal of Urology, 7, 103-112.

https://doi.org/10.4236/oju.2017.77013

Received: May 31, 2017

Accepted: July 15, 2017

Published: July 18, 2017

Copyright $\odot 2017$ by authors and Scientific Research Publishing Inc. This work is licensed under the Creative Commons Attribution International License (CC BY 4.0).

http://creativecommons.org/licenses/by/4.0/ (c) (i) Open Access

\begin{abstract}
Objectives: To evaluate the efficacy of intranasal desmopressin compared with intramuscular diclofenac and combination of both in the treatment of acute renal pain caused by stone disease. Patients and methods: Ninety patients (51 males and 39 females) presented to our hospital emergency department (Al-Yarmouk Teaching Hospital/Baghdad/Iraq) with the diagnosis of acute renal pain caused by urolithiasis. They were randomized into three equal groups; group A received desmopressin $40 \mu \mathrm{g}$ intranasally (4 puffs, each puff equivalent to 10 micrograms), group $B$ received intramuscular diclofenac $75 \mathrm{mg}$ and group $\mathrm{C}$ received both desmopressin and diclofenac. A visual analogue scale was used to assess the pain intensity in the 3 groups at baseline, 10, 20 and 30 min after drugs administration. Results: At presentation, the pain intensity was similar in all three groups. For patients in group $\mathrm{A}$, the initial pain score was 9.5 then it declined to 5.2, 3.7 and 3.7 at 10, 20 and 30 minutes after administration of desmopressin, and for patients in group B, the initial pain score was 9.8 then became 4.6, 2.9 and 2.3 at 10, 20 and 30 minutes after diclofenac, while for patients in group $\mathrm{C}$, the initial pain score was 9.7 then became 4.8, 2.6 and 2.2 at 10, 20 and 30 minutes after administration of desmopressin and diclofenac. Conclusion: $40 \mu \mathrm{g}$ intranasal desmopressin sprays can be used to relieve pain in patients with acute renal pain either alone or combined with diclofenac.
\end{abstract}

\section{Keywords}

Renal Pain, Desmopressin, Diclofenac 


\section{Introduction}

Pain is the most common complaint of emergency department patients seen in one half to three quarters of all patients [1] [2]. Renal colic is a common presentation (lifetime risk $12 \%$ in men, $6 \%$ in women) [3].

Renal pain is caused by an increase in pelvi-ureteric pressure secondary to an obstruction of the urinary tract. This increase in pressure causes a prostaglandin (PG)-mediated increase in renal blood flow and a subsequent increase in diuresis which, in turn, further increases intrapelvic pressure and results in more pain [4] [5]. Modulation of antidiuretic hormone (ADH) is probably one of the most important mechanisms leading to an increased diuresis and one of the roles of PGs seems to be blocking the action of ADH by interfering with cAMP-mediated signal transmission. Accordingly it would be expected that the level of ADH may influence the outcome of renal pain therapy [4].

Many medications are used for relieving renal colic pain \& helping the passage of renal stone: narcotics \& opiates, non-steroidal anti-inflammatory drugs (NSAIDs), antidiuretics, alpha blockers, Ca channel blockers, steroids \& anticholinergics [6].

NSAIDs (inhibitors of prostaglandin synthesis with Diclofenac being the most appropriate) have long been used as effective agents in the treatment of renal pain. They are the first-line treatment for renal colic pain because they have been shown to achieve greater reductions in pain scores, have a longer duration of action and result in a reduced need for additional analgesia in the short-term compared with patients treated with opioid analgesics [7].

Desmopressin [1-desamino-8-D-arginine Vasopressin, DDAVP] is a synthetic vasopressin analogue. Compared to vasopressin, desmopressin possesses enhanced antidiuretic effect, prolonged duration of action and less vasopressor activity [8].

The mechanism of analgesic action of desmopressin in renal pain is uncertain. At the peripheral level, desmopressin may alleviate the acute renal colic through its potent antidiuretic effect or by relaxing the renal pelvic and ureteral smooth muscles. The central analgesic effect of desmopressin by stimulating the release of the hypothalamic $\beta$-endorphins is proposed [9] [10].

Our study was designed to determine the effect of intranasal desmopressin spray on acute renal colic pain and compare it with the effect of intramuscular diclofenac sodium or combination of both.

\section{Patients and Methods}

This prospective study was conducted between $24^{\text {th }}$ October 2015 and $2^{\text {nd }}$ August 2016 and included 90 patients (51 males and 39 females) with mean age 33.9 years presented to our hospital emergency department (Al-Yarmouk Teaching Hospital/Baghdad/Iraq) with the diagnosis of acute renal pain caused by urolithiasis.

The diagnosis of renal colic was based on full history taking including the severity, site $\&$ the duration of pain at presentation, the associated symptoms such 
as hematuria and symptoms of urinary tract infection, previous episodes of renal pain, past medical and surgical history and history of medications.

Renal pain intensity at presentation was assessed \& recorded using a $10 \mathrm{~cm}$ visual analogue scale (VAS) graded in 10 equal intervals ranging from 0 (no pain) to 10 (unbearable pain) as shown in Figure 1. It was explained to the patients how to use this scale and was asked to point to the mark that represented the pain intensity after each interval in the assessment.

Vital signs and positive findings of the physical examination were evaluated and recorded.

Basic investigations were performed including urinalysis, full blood count, serum electrolytes, blood urea and serum creatinine, KUB and abdominal and pelvic ultrasound.

All patients with acute renal pain caused by urolithiasis and had previously received no treatment were included in the study while patients with arterial hypertension, coronary artery disease, a common cold or rhinitis, coagulopathy, anticoagulant therapy, peptic ulcer, azotemia or liver failure \& pregnancy were excluded from the study.

The patients were randomized into three equal groups, group A received desmopressin $40 \mu \mathrm{g}$ intranasally (4 puffs, each puff equivalent to 10 micrograms \& alternately in each nostril of the nose), group B received intramuscular diclofenac $75 \mathrm{mg}$ and group $\mathrm{C}$ received both desmopressin and diclofenac, $40 \mu \mathrm{g}$ inranasal desmopressin was administered about five minutes before $75 \mathrm{mg}$ IMdiclofenac. We used the combination in this group to see if there was any difference in the effect on pain relief.

Renal pain was reassessed using the visual analogue scale at 10, 20 and $30 \mathrm{mi}-$ nutes after drug administration.

In patients who had no satisfactory pain relief at 30 minutes a second treatment was given according to the following pre-established protocol: group A received intramuscular diclofenac $75 \mathrm{mg}$, group B received intranasal desmopressin $40 \mu \mathrm{g}$ and group $\mathrm{C}$ received Tramadol or Pethidine. Any adverse reaction was recorded. The results were assessed statistically.

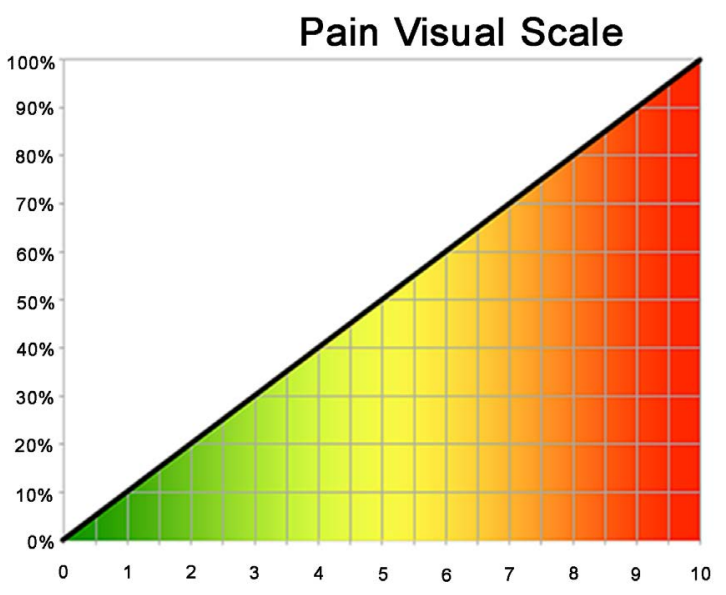

Figure 1. Pain visual analogue scale. 


\section{Results}

A total of 90 patients with acute renal colic pain were evaluated in this study. Twenty five patients had a history of stone disease; 20 passed their stones per urethra within the last 8 years while 5 underwent percutaneous nephrolithotomy in 3 patients \& open surgery in 2 patients within the last 6 months (Table 1).

The mean number of previous episodes was $1.9(2.2,1.94 \& 1.6$ in group A, B $\& \mathrm{C}$ respectively).

After the random assignment, each group included 30 patients. our 90 patients were distributed according to age, sex, site and duration of pain as follows; the distribution of the patients according to age was 6 patients $(7 \%)<20$ years, 29 patients (32\%) 20 - 29 years, 25 patients (28\%) 30 - 39 years, 19 patients (21\%) 40 - 49 years and 11 patients $(12 \%) \geq 50$ years as shown in Table 2 \& Figure 2 \& Figure 3.

The distribution of patients according to sex was 51 (57\%) males and $39(43 \%)$ females as shown in Figure 4 \& Figure 5.

Table 1. Patients with history of stone disease.

\begin{tabular}{cc}
\hline History of stone disease & No. (\%) \\
\hline Spontaneous passage & $20(80 \%)$ \\
PCNL & $3(12 \%)$ \\
Open surgery & $2(8 \%)$ \\
Total & $25(100 \%)$ \\
\hline
\end{tabular}

Table 2. The demographic characteristics of the patients.

\begin{tabular}{cc}
\hline Gender & $57 \%$ males $43 \%$ females \\
\hline Patient's age & No. (\%) \\
\hline$<20$ years & $6(7 \%)$ \\
$20-29$ years & $29(32 \%)$ \\
$30-39$ years & $25(28 \%)$ \\
$40-49$ years & $19(21 \%)$ \\
$\geq 50$ years & $11(12 \%)$ \\
Total & $90(100 \%)$ \\
\hline
\end{tabular}

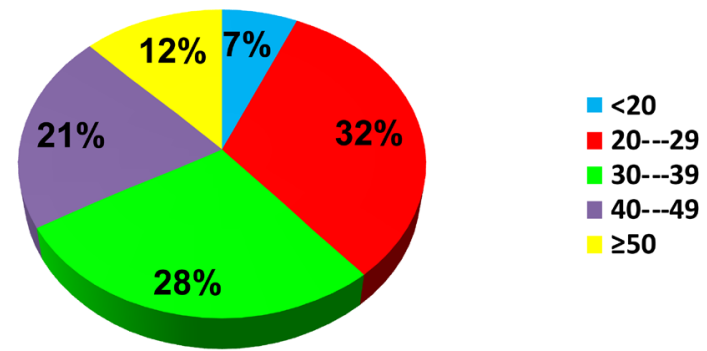

Figure 2. Distribution of patients according to age (years). 


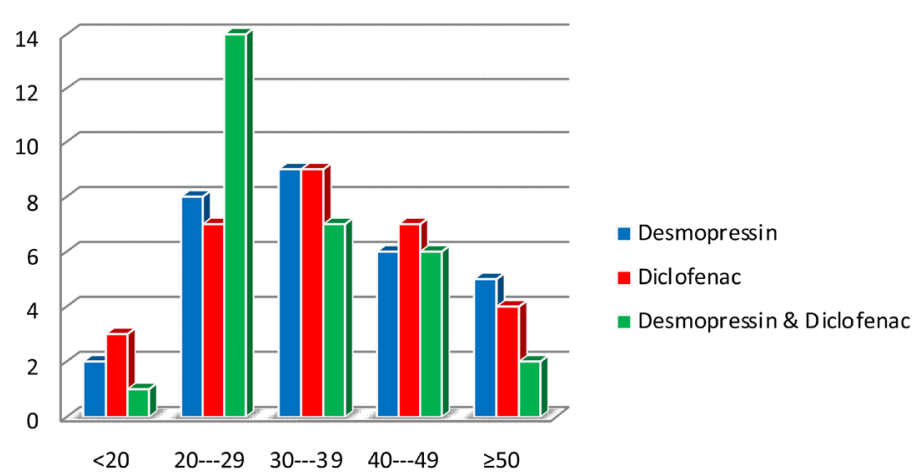

Figure 3. The distribution of patients according to age (years) in the three groups.

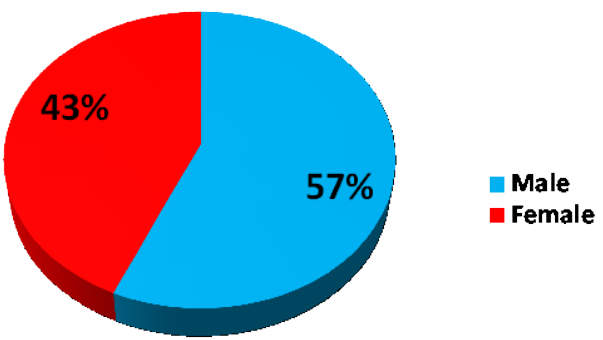

Figure 4. Distribution of patients according to sex.

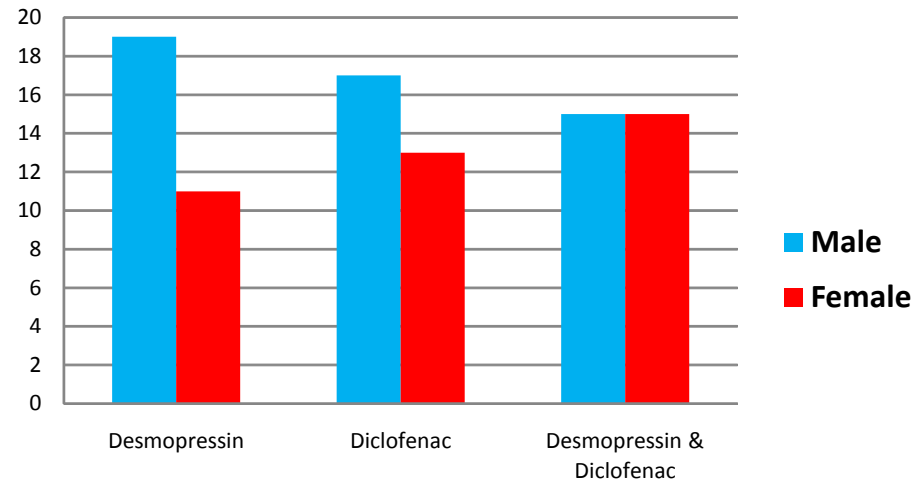

Figure 5. The distribution of patients according to sex in the three groups.

While Figure 6, Figure 7 showed the distribution of patients according to site of pain which was 33 patients (37\%) in the right side, 52 patients $(58 \%)$ in the left side and 5 patients (5\%) with bilateral pain.

And the distribution of patients according to duration of pain (hours) was 53 patients (59\%) less than 6 hours and 37 patients (41\%) more than 6 hours as shown in Figure 8 \& Figure 9.

It seems that the effect of age, sex, site, and duration of pain was statistically insignificant in the results of our study (the Pearson Chi-square value for all these variables was more than 0.05) (Table 3).

At presentation all patients had nearly the same pain visual analogue score reaching 10. After 10 minutes the mean pain score was 5.2 in group A, 4.6 in 


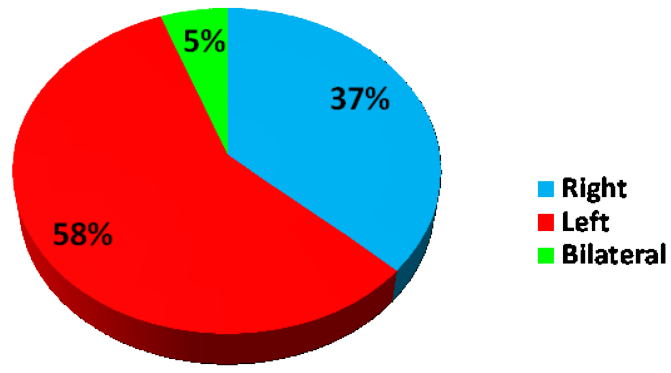

Figure 6. The distribution of patients according to site of pain.

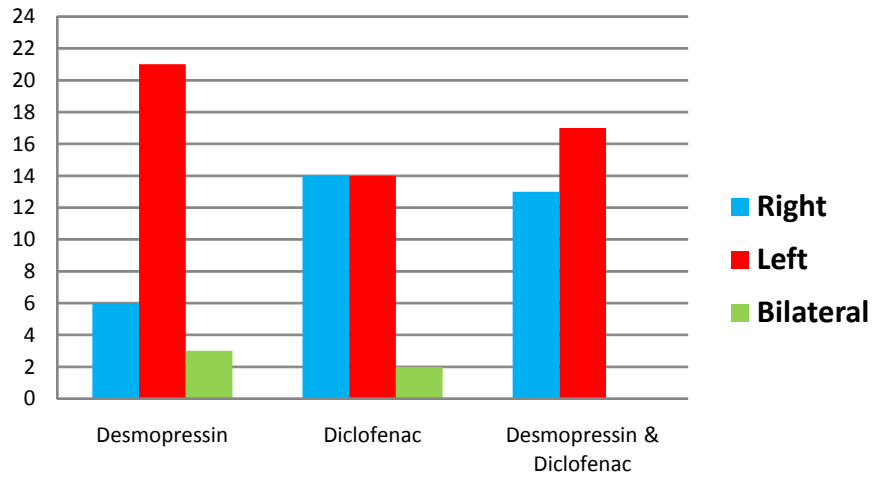

Figure 7. The distribution of patients according to site of pain in the three groups.
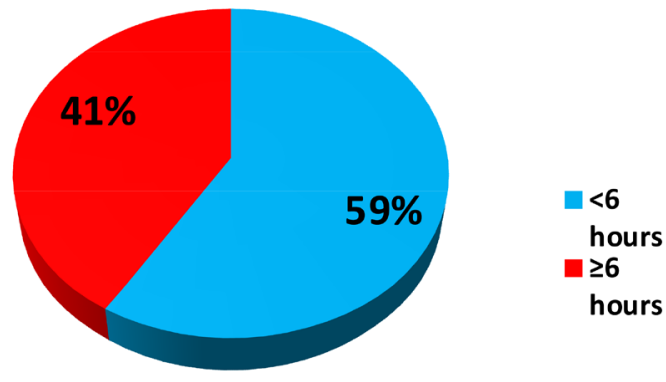

Figure 8. The distribution of patients according to duration of pain (hours).

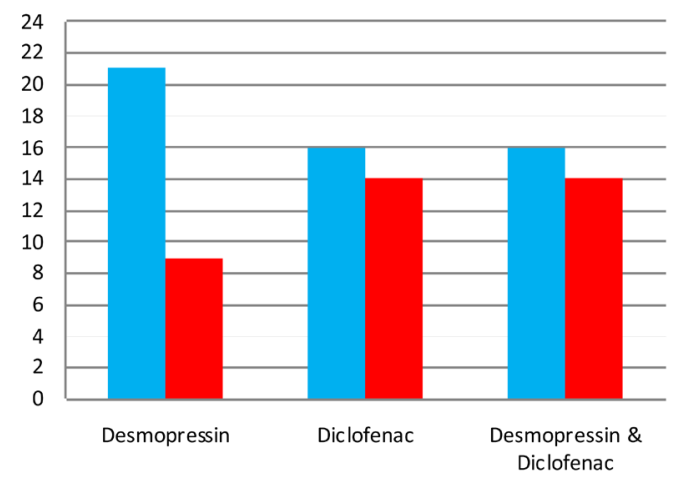

Figure 9. The distribution of patients according to the duration of pain (hours) in the three groups. 
Table 3. The effects of age, sex, site and duration of pain in the three groups.

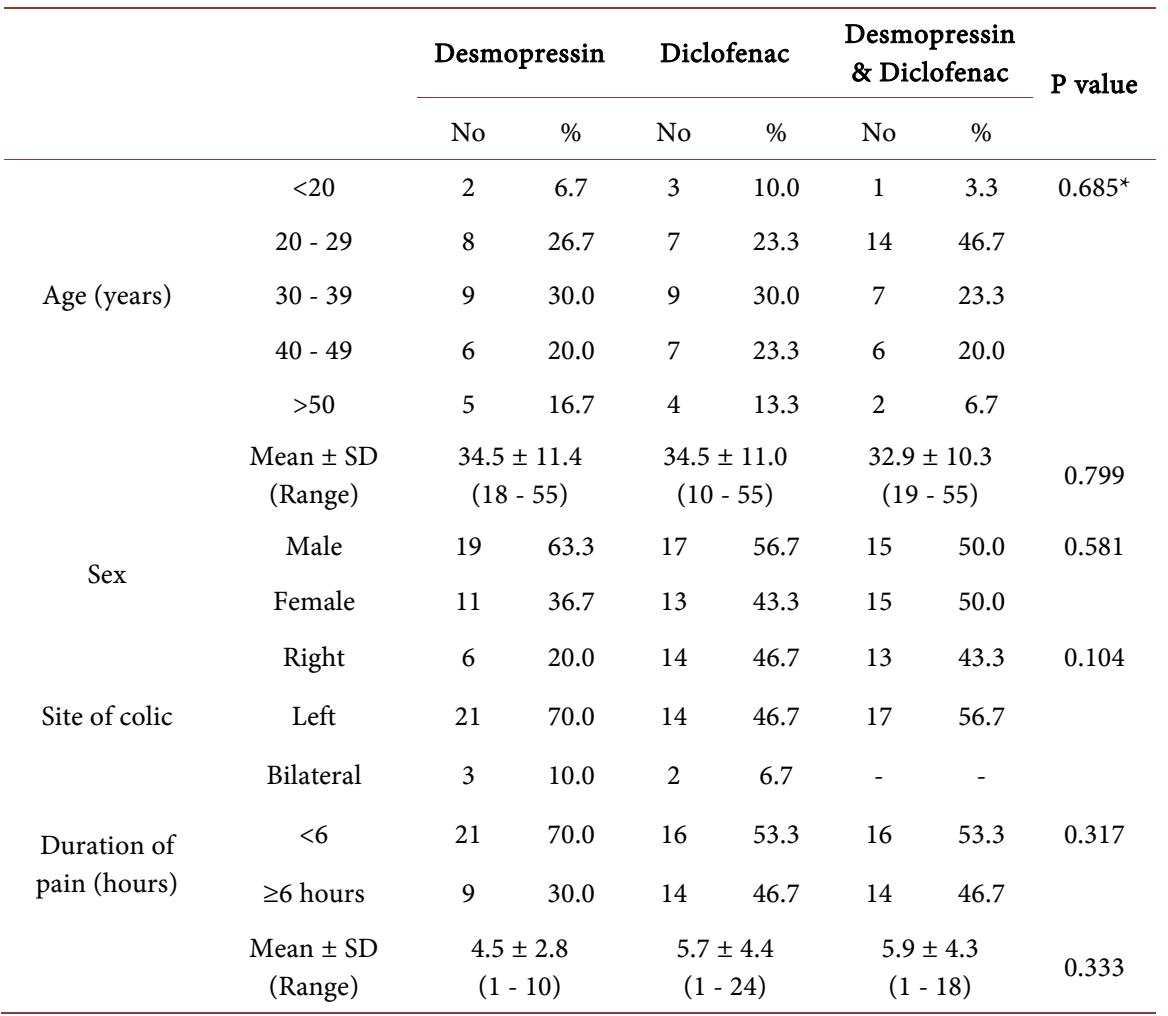

${ }^{*}$ Significant difference between proportions using Pearson Chi-square test at 0.05 level or less.

group B and 4.8 in group C. After 20 minutes the mean pain score was 3.7 in group A, 2.9 in group $B$ and 2.6 in group $C$, and after 30 minutes the mean pain score was 3.7 in group A, 2.3 in group B and 2.2 in group C., when combination of desmopressin and diclofenac was administered we experienced that the best improvement in pain score was achieved when desmopressin was administered about five minutes before diclofenac.

In general, the improvement in mean pain score was less with desmopressin than with diclofenac or combination of both as shown in Figure 10.

13 patients $(43.3 \%)$ in group A needed a second treatment with diclofenac intramuscularly. In group B 5 patients (16.6\%) required a second treatment with intranasal desmopressin while only 4 patients $(13.3 \%)$ in group C needed a second treatment with tramadol/pethidine.

Of the patients $27.7 \%$ had an associated UTI. Side effects were reported in 6 patients (6.6\%); in group A two patients had a dry mouth, in group B two patients had vomiting while in group $\mathrm{C}$ one patient had vomiting \& the other one had postural hypotension.

\section{Discussion}

NSAIDs remain the mainstay treatment of acute renal pain; however, desmopressin can be used as alternative to NSAIDs in some situations and can also be used as adjuvant treatment with NSAIDs. 


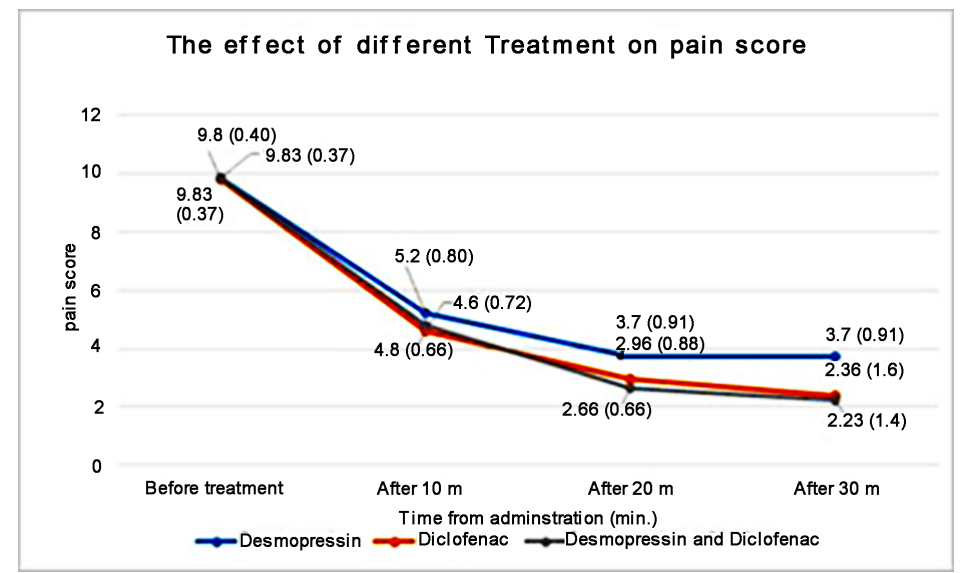

Figure 10. The effect of desmopressin, diclofenac \& their combination on mean pain score $+(\mathrm{SD})$.

In our study, for patients in group A , the initial pain score was 9.5 then it declines to 5.2, 3.7 and 3.7 at 10, 20 and 30 minutes after administration of desmopressin, and for patients in group B, the initial pain score was 9.8 then it declines to $4,6,2.9$ and 2.3 at 10,20 and 30 minutes after administration of diclofenac, while for patients in group $C$, the initial pain score was 9.7 then it declines to 4 , $8,2.6$ and 2.2 at 10, 20 and 30 minutes after administration of desmopressin and diclofenac.

This finding strongly suggested that the administration of $40 \mu \mathrm{g}$ intranasal desmopressin spray resulted in a prompt decrease in renal pain intensity at 30 minutes of administration taking into consideration the slow absorption of this drug from the nasal mucosa $\&$ assuming a further decrease in pain intensity if the assessment continued beyond 30 minutes reflecting the progressive intranasal absorption of desmopressin [11].

Our study demonstrated that patients who received intranasal desmopressin \& continued complaining of pain, required a second treatment with $75 \mathrm{mg}$ diclofenac sodium IM to relieve their pain. This result suggested that the pain relieving effect of intramuscular diclofenac was enhanced by the prior administration of intranasal desmopressin potentiating each drug's analgesic effect, a finding which was in accordance with El-Sherif et al. [8] \& Grenabo et al. [10] who stated that NSAID action is more effective in the presence of higher plasma levels of $\mathrm{ADH}$.

The mechanism by which desmopressin relieves acute renal colic pain was described in the introduction [9] [10].

Other variables such as, age, sex, site and duration of pain had no significant impact on the action of desmopressin on renal pain.

Our results were consistent with that obtained by Lopes et al. 2001 [4] and Ibrahim A. M 2006 [12] who had studied the analgesic effect of intranasal desmopressin on renal pain compared with intramuscular diclofenac and their combination.

However our study was different from that of Masoumi, K. et al. 2014 [3] who compared analgesic effect of $75 \mathrm{mg}$ intramuscular sodium diclofenac and $40 \mu \mathrm{g}$ 
intranasal desmopressin combination (group A) with intramuscular sodiumdiclofenac alone (group B) in patients with acute renal colic. The pain score of patients was assessed using a visual analogue scale (VAS) at baseline, 15, 30, 45, and 60 minutes after administration In this study, the baseline VAS score was not significantly different between two groups. The Mean \pm SD scores of two groups reduced 15 minutes after drug administration, but this decrease was significantly more in Group A compared with Group B. This pattern continued in minutes 30,45 , and 60 of drug administration. This study showed that desmopressin could be used as an effective adjuvant in acute renal colic pain management.

About 15 patients were followed up for two weeks ( 5 in each group), we found that the type of analgesia had no impact on eventual stone passage.

\section{Conclusion}

Desmopressin intranasal spray is a safe drug, easy to administer, well tolerated \& effective in treating acute renal pain either alone or combined with other analgesic medications like diclofenac.

\section{References}

[1] Fosnocht, D.E., Swanson, E.R. and Bossert, P. (2001) Patient Expectations for Pain Medication Delivery. American Journal of Emergency Medicine, 19, 399.

https://doi.org/10.1053/ajem.2001.24462

[2] Weisman, S.J., Bernstein, B. and Schechter, N.L. (1998) Consequences of Inadequate Analgesia during Painful Procedures in Children. Archives of Pediatrics and Adolescent Medicine, 152, 147. https://doi.org/10.1001/archpedi.152.2.147

[3] Masoumi, K., Darian, A.S., Forouzan, A., et al. (2014) The Efficacy of Intranasal Desmopressin as an Adjuvant in the Acute Renal Colic Pain Management. Pain Research and Treatment 2014, Article ID 320327, 5. https://doi.org/10.1155/2014/320327

[4] Lopes, T., Dias, J.S., Marcelino, J., et al. (2001) An Assessment of the Clinical Efficacy of Intranasal Desmopressin Spray in the Treatment of Renal Colic. BJU International, 87, 322-325. https://doi.org/10.1046/j.1464-410x.2001.00068.x

[5] Conradie, M.C. (2012) Management of Renal Colic and Triage in the Emergency Room. In: Smith, A.D., Badlani, G.H., Preminger, G.M. and Kavoussi, L.R., Eds., Smith Textbook of Endourology. Wiley Blackwell, Chichester, 675-687. https://doi.org/10.1002/9781444345148.ch59

[6] Beladi, S.S.M., Darian, A.S., Forouzan, A., et al. (2012) Renal Colic Pain Relief by Intranasal Desmopressin. Life Science Journal, 9, 3354-3358.

[7] Heid, F. and Jage, J. (2002) The Treatment of Pain in Urology. BJU International, 90, 481-488. https://doi.org/10.1046/j.1464-410X.2002.02908.x

[8] El-Sherif, A.E., Salem, M., Yahya, H., et al. (1996) Treatment of Renal Colic by Desmopressin Intranasal Spray and Diclofenac Sodium. The Journal of Urology, 153, 1395-1398. https://doi.org/10.1016/S0022-5347(01)67411-1

[9] Roshani, A., Falahatkar, S., Khosropanah, I., et al. (2010) Assessment of Clinical Efficacy of Intranasal Desmopressin Spray and Diclofenac Sodium Suppository in Treatment of Renal Colic versus Diclofenac Sodium Alone. Urology, 75, 540-542. 
https://doi.org/10.1016/j.urology.2008.05.053

[10] Grenabo, L., Aurell, M., Delin, K., et al. (1983) Antidiuretic Hormone Levels and the Effect of Indomethacin on Ureteral Colic. The Journal of Urology, 129, 941-943. https://doi.org/10.1016/S0022-5347(17)52468-4

[11] Seif, S.M., Zenser, T.V., Clarochi, F.F., et al. (1978) DDAVP (1-Desamino8-D-Argenine-Vasopressin) Treatment of Central Diabetes Incipidus-Mechanism of Prolonged Antidiuresis. The Journal of Clinical Endocrinology \& Metabolism, 46, 381-388. https://doi.org/10.1210/jcem-46-3-381

[12] Ibrahim, A.M. (2007) Evaluation of Using Intranasal Desmopressin, Parenteral Diclofenac or Their Combination in the Management of Acute Renal Colic Pain in Iraqi Patients. Iraqi Journal of Pharmaceutical Sciences, 16, 1-4

Submit or recommend next manuscript to SCIRP and we will provide best service for you:

Accepting pre-submission inquiries through Email, Facebook, LinkedIn, Twitter, etc. A wide selection of journals (inclusive of 9 subjects, more than 200 journals)

Providing 24-hour high-quality service

User-friendly online submission system

Fair and swift peer-review system

Efficient typesetting and proofreading procedure

Display of the result of downloads and visits, as well as the number of cited articles

Maximum dissemination of your research work

Submit your manuscript at: http://papersubmission.scirp.org/

Or contactoju@scirp.org 